\title{
miR-224 functions as an onco-miRNA in hepatocellular carcinoma cells by activating AKT signaling
}

\author{
DONGLAI MA, XUANCHEN TAO, FENG GAO, CHENGJUAN FAN and DEQUAN WU \\ Department of General Surgery, The Second Affliated Hospital of Harbin Medical University, \\ Harbin, Heilongjiang 150086, P.R. China
}

Received February 28, 2012; Accepted May 31, 2012

DOI: $10.3892 / \mathrm{ol} .2012 .742$

\begin{abstract}
RNAs (miRNAs) are a class of small non-coding RNAs that post-transcriptionally regulate gene expression. Increasing evidence has shown that the deregulation of miRNAs is linked to cancer. The overexpression of miR-224 has been reported in several human cancers. The aim of the present study was to investigate the function of miR-224 in the pathogenetic process of hepatocellular carcinoma (HCC), and the precise mechanism underlying its function. Both gain-of-function and loss-of function assays were conducted through transfection with miR-224 mimics and miR-224 inhibitors in the HepG2 liver carcinoma cell line. The data revealed that miR-224 exerts a significant role in promoting cell proliferation, migration and invasion. Western blot analysis showed that the phosphorylation levels of AKT positively correlated with endogenous levels of miR-224. In addition, results from a dual luciferase reporter assay showed that the expression of the serine/threonine-protein phosphatase $2 \mathrm{~A} 65 \mathrm{kDa}$ regulatory subunit $\mathrm{A} \beta$ isoform (PPP2R1B) is inhibited by miR-224; thus, it appears that PPP2R1B is a candidate target of miR-224 in HCC. These data suggest that miR-224 plays a significant role in HCC, possibly through the activation of the AKT signaling pathway by targeting PPP2R1B.
\end{abstract}

\section{Introduction}

Hepatocellular carcinoma (HCC) is the seventh most prevalent cancer worldwide, accounting for $85-90 \%$ of all primary liver cancers, and ranking as the fourth leading cause of cancer-related mortality $(1,2)$. Despite great advances in the treatment of $\mathrm{HCC}$, the 5-year survival rate remains poor. In

Correspondence to: Dr Dequan Wu, Department of General Surgery, The Second Affliated Hospital of Harbin Medical University, 194 Xue Fu Road, Harbin, Heilongjiang 150086, P.R. China

E-mail: dequanwu@126.com

Key words: miR-224, hepatocellular carcinoma, PPP2R1B, AKT signaling order to reduce the high mortality rate, it is critical to clarify the precise mechanism underlying HCC development and progression.

miRNAs are small non-coding RNAs of approximately 19-25 nucleotides that post-transcriptionally regulate gene expression. miRNAs usually bind to the 3 ' untranslated region (UTR) of target mRNAs through their seed sequences, leading to the suppression of translation, and occasionally mRNA degradation $(3,4)$. miRNAs are estimated to modulate $30 \%$ of human protein-coding genes (5). Increasing evidence has shown that miRNAs have significant roles in diverse biological and pathological processes (6). Meanwhile, the deregulation of miRNAs has been observed in diverse diseases, including cancer $(7,8)$. Numerous studies have shown that miRNAs are frequently deregulated in HCC. For example, miR-21 is upregulated in HCC and negatively regulates the expression of the tumor suppressor gene, phosphatase and tensin homolog (PTEN), which promotes cancer progression and metastasis (9). Wong et al suggested that miR-222 functions as a metastatic activator in HCC via the activation of the AKT signaling pathway by targeting PPP2R2A (10). By contrast, certain metastatic suppressive miRNAs including miR-34a, miR-23b, miR-122 and miR-124 are frequently downregulated in HCC and facilitate tumor metastasis by regulating vital genes (11-14).

The abnormal expression of miR-224 has been reported in several human cancers (15-19). Wang et al found that miR-224 is upregulated in HCC patients and HCC cell lines (20), and promotes HCC cell apoptosis by targeting the transcript expression levels of apoptosis inhibitor 5 (API-5). Simultaneously, miR-224 also promotes HCC cell proliferation, although its potential target gene is currently unknown. More recently, miR-224 has been confirmed to be involved in the malignant phenotype of HepG2 cells, and is reported to be a significant factor in the regulation of the migration and invasion of HepG2 cells (21); however, the authors did not propose a potential target gene that may serve as an intermediary between miR-224 and metastasis in HCC cells.

In the present study, TargetScan, PicTar and miRBase Targets were employed to predict the putative targets of miR-224, which has a pivotal role in cell proliferation and metastasis. We selected the serine/threonine-protein phosphatase $2 \mathrm{~A} 65 \mathrm{kDa}$ regulatory subunit $\mathrm{A} \beta$ isoform (PPP2R1B) as a target for our study, since PP2A is a well-conserved and essen- 
tial protein serine/threonine phosphatase, as well as a critical regulator in the control of certain key proteins of oncogenic signaling cascades (22). It has also been suggested that PP2A has an effect on the regulation of motility and invasion of both normal and transformed cells (23). Hamano et al demonstrated that miR-200c induces chemoresistance in esophageal cancer through the activation of the AKT signaling pathway, and the authors proposed that miR-200c stimulates the AKT signaling pathway by specifically targeting PPP2R1B (24). Therefore, we postulated that miR-224 might impact the proliferation and metastasis of HCC cells through the activation of the AKT signaling pathway by targeting the PPP2R1B tumor suppressor gene.

\section{Materials and methods}

Cell culture. The human HCC cell line HepG2 was purchased from the Shanghai Institute of Cell Biology (Academia Sinica, Shanghai, China). Cells were cultured in Dulbecco's modified Eagle's medium (DMEM) high glucose supplemented with heat-inactivated $10 \% \mathrm{FBS}, 100 \mathrm{U} / \mathrm{ml}$ penicillin and $100 \mu \mathrm{g} / \mathrm{ml}$ streptomycin at $37^{\circ} \mathrm{C}$ in a humidified incubator containing $5 \%$ $\mathrm{CO}_{2}$.

Analysis of miR-224 expression by $q R T-P C R$. Reverse transcription and quantitative real-time PCR (qPCR) were employed to measure the expression levels of miR-224 in HepG2 cells. Total RNA was isolated using TRIzol reagent (Invitrogen, Carlsbad, CA, USA). Whole-cell cDNA was synthesized, then miR-224 expression was analyzed using a real-time PCR instrument (ABI7000) and an All-in-One ${ }^{\mathrm{TM}}$ miRNA qRT-PCR detection kit (GeneCopoeia, Rockville, $\mathrm{MD}, \mathrm{USA}$ ). The reverse transcription reaction parameters were $37^{\circ} \mathrm{C}$ for $60 \mathrm{~min}$ followed by $85^{\circ} \mathrm{C}$ for $5 \mathrm{~min}$. The PCR parameters were as follows: $95^{\circ} \mathrm{C}$ for $10 \mathrm{~min}$, followed by 40 cycles of $95^{\circ} \mathrm{C}$ for $10 \mathrm{sec}, 60^{\circ} \mathrm{C}$ for $20 \mathrm{sec}$, and $72^{\circ} \mathrm{C}$ for $3 \mathrm{sec}$. The expression levels of miR-224 were normalized to RNU6B levels. All reactions were performed in triplicate and included negative control reactions without cDNA.

miRNAs and transfection. The miRNAs were designed and synthesized by GenePharma (Shanghai, China). The following Homo sapiens (has)-miR-224 mimics were synthesized: sense, 5'-CAA GUC ACU AGU GGU UCC GUU-3'; antisense, 5'-CGG AAC CAC UAG UGA CUU GUU-3'; negative control: sense, 5'-UUC UCC GAA CGU GUC ACG UTT-3'; antisense, 5'-ACG UGA CAC GUU CGG AGA ATT-3'; has-miR-224 inhibitor: 5'-AAC GGA ACC ACU AGU GAC UUG-3'; has-miR-224 inhibitor negative control: 5'-CAG UAC UUU UGU GUA GUA CAA-3'. miRNA transfection was performed using FuGENE HD transfection reagent (Indianapolis, IN, USA). In brief, $4 \times 10^{5}$ cells were plated in $2 \mathrm{ml}$ medium in the wells of a six-well plate. Cells were incubated overnight to achieve $>80 \%$ confluence at the time of transfection. In each well, $5 \mu 1$ miRNA was added to $100 \mu 1$ serum-free medium. Separately, $5 \mu$ l FuGENE HD transfection reagent was added to $100 \mu \mathrm{l}$ serum-free medium and mixed gently. The transfection complex was incubated for $15 \mathrm{~min}$ at room temperature, added to cells, and incubated for $6 \mathrm{~h}$ after which the serumcontaining medium was replaced. Following transfection, the cells were incubated for 48 and $72 \mathrm{~h}$ prior to qPCR and western blot analysis, respectively.

Western blot analysis. Total protein from HepG2 cells was extracted with 1\% RIPA lysis buffer (Beyotime, Shanghai, China) containing $1 \mathrm{mM}$ phenylmethanesulfonylfluoride (PMSF). The supernatants were collected, and protein concentration was determined using the BCA assay kit (Beyotime). Proteins were resolved on $12 \%$ SDS-PAGE gels using a minigel apparatus (Bio-Rad Laboratories, Hercules, CA, USA) and transferred to nitrocellulose membranes. The membranes were blocked for $1 \mathrm{~h}$ at room temperature and incubated overnight at $4^{\circ} \mathrm{C}$ with primary antibody, followed by incubation with HRP-conjugated secondary antibody after three 10-min washes in TBST. After incubation with secondary antibody, the membrane was washed three times for $10 \mathrm{~min}$ in TBST, and the protein was detected by chemiluminescence with the ECL detection reagent (Pierce Biotechnology, Inc., Rockford, IL, USA). The data were normalized to $\beta$-actin.

Luciferase reporter assay. Human PPP2R1B 3'UTR containing the miR-224 target sequence (position 141-147) and mutant sequences were chemically synthesized (GenScript, Nanjing, China) and cloned into the PGL3 control vector (Promega, Madison, WI, USA) downstream of the luciferase gene using the $X b a \mathrm{I}$ site. The resulting plasmids were designated pPPP2R1B 3'UTR-luci-WT and pPPP2R1B 3'UTR-luci-MUT, respectively. HepG2 cells were transfected with either pPPP2R1B 3'UTR-luci-WT or pPPP2R1B 3'UTR-luci-MUT in 24-well plates using FuGENE HD transfection reagent according to the manufacturer's instructions, and co-transfected with either $30 \mathrm{nM}$ of has-miR-224 mimics (GenePharma) or the same concentration of negative control (GenePharma). Each well was also co-transfected wth $50 \mathrm{ng}$ of the pRL-TK plasmid (Promega) to determine transfection efficiency. Then, firefly and Renilla luciferase activity levels were measured in cells that were harvested $24 \mathrm{~h}$ post-transfection, using the DualLuciferase Reporter assay kit (Promega). Each transfection was performed in triplicate.

Cell proliferation assay. The viability of cells was determined using the Cell Counting Kit-8 (Dojindo Laboratories, Japan). Cells were typsinized and seeded into 96-well plates at a density of $5 \times 10^{3}$ cells/well $6 \mathrm{~h}$ after transfection. After incubation in a humidified incubator $\left(37^{\circ} \mathrm{C}, 5 \% \mathrm{CO}_{2}\right)$ for different time periods, $10 \mu \mathrm{l} \mathrm{CCK}-8$ was added to each well, followed by incubation at $37^{\circ} \mathrm{C}$ for another $1 \mathrm{~h}$. The absorbance was measured at $450 \mathrm{~nm}$ using a microplate reader to evaluate the number of viable cells.

Transwell migration and invasion assays. The Transwell chamber with $8-\mu \mathrm{m}$ pore filters $(6.5 \mathrm{~mm}$ in diameter, $8 \mu \mathrm{m}$ pore size, Corning Inc., Lowell, MA, USA) was used for the migration and invasion assays. For the migration assay, HepG2 cells were transfected with the desired miRNAs, and then trypsinized $48 \mathrm{~h}$ after transfection. The cells were then resuspended in serum-free medium, and the concentration was adjusted to $1 \times 10^{5}$, followed by the addition of $200 \mu 1$ cell suspension to the upper chamber. Next, $700 \mu 1$ medium containing $20 \%$ FBS was added to the lower chamber. Cells 
A

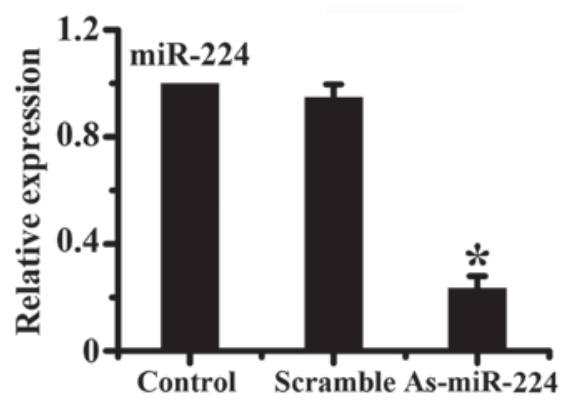

C

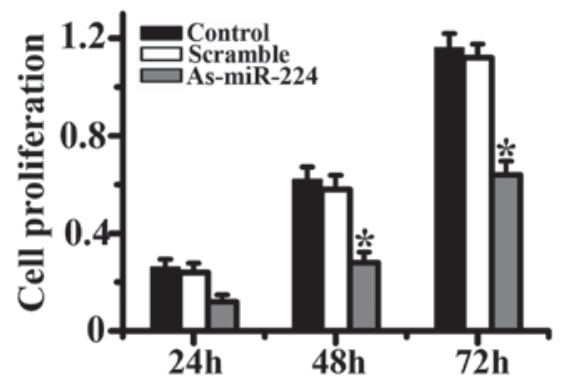

B

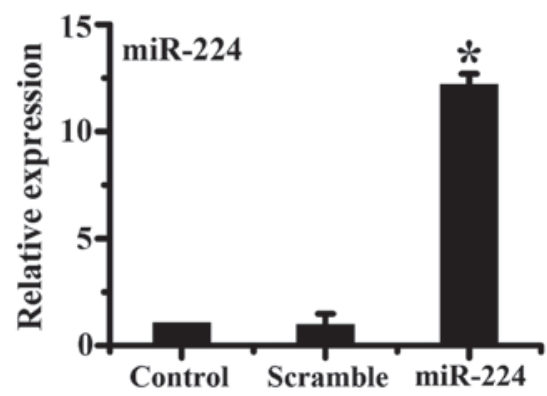

D

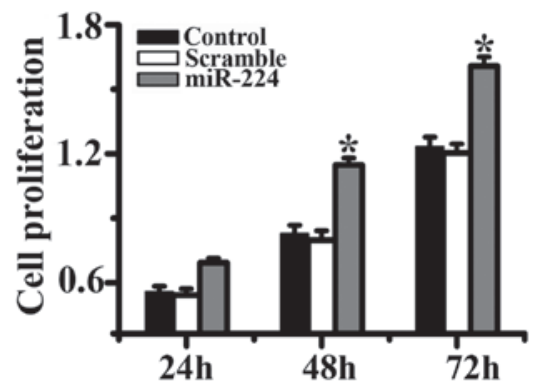

Figure 1. miR-224 regulates HepG2 cell proliferation. Semi-quantitative real-time PCR was performed to measure miR-224. (A) The expression level of miR-224 (normalized to U6 RNA) was significantly decreased by $85 \%$ in the miR-224 inhibitor group relative to the scrambled group; (B) miR-224 was notably upregulated by $\sim 14$ times in HepG2 cells treated with miR-224 mimics compared to scrambled oligonucleotides. Viable cells were detected by the CCK-8 assay 24, 48 and $72 \mathrm{~h}$ after transfection; (C) Cell proliferation was markedly suppressed by downregulation of miR-224 levels compared to the controls; (D) Viable cells in the miR-224 mimic group were significantly higher than in the control groups. Data are expressed as the mean of three separate experiments \pm SD. ${ }^{*} \mathrm{P}<0.05$. As-miR-224, antisense miR-224.

were incubated for $24 \mathrm{~h}$ at $37^{\circ} \mathrm{C}$ in a humidified incubator containing $5 \% \mathrm{CO}_{2}$. Then, cells that migrated to the bottom of the membrane were fixed in $4 \%$ paraformaldehyde and stained with crystal violet for $10 \mathrm{~min}$ at room temperature, followed by visualization under a microscope after washing with distilled water. For the invasion assay, the protocol was the same as the migration assay, except that the upper chamber was precoated with $1 \mathrm{mg} / \mathrm{ml}$ Matrigel (BD Biosciences, Bedford, MA, USA), followed by prolonged incubation for $36 \mathrm{~h}$.

Statistical analysis. All data are presented as the mean \pm standard deviation (SD) from at least three separate experiments. Differences among experimental groups were analyzed by the Student's t-test using the SPSS Graduate Pack statistical software, version 11.0. $\mathrm{P}<0.05$ was considered to indicate a statistically significant result.

\section{Results}

miR-224 positively regulates HCC cell proliferation in vitro. To assess the role of miR-224 in the proliferation of HCC cells, both loss-of-function and gain-of-function approaches were employed in our study. HepG2 cells were treated with miR-224 mimics or miR-224 inhibitors and control scramble oligonucleotides. Results from qPCR demonstrated that the relative expression of miR-224 was successfully modulated in both the miR-224 mimic and miR-224 inhibitor groups compared with the control group. Cell proliferation was evaluated by the CCK-8 assay at different time points. The results showed that the downregulation of miR-224 significantly inhibited cell proliferation compared with the control group, while relative to the control group, the upregulation of miR-224 significantly enhanced the cell proliferation capability of HCC cells (Fig. 1).

Cell migration and invasion promoted by miR-224. In order to determine the effect of miR-224 on cell migration and invasion, HepG2 cells were transfected with synthetic oligonucleotides and divided into four groups: miR-224 mimics, miR-224 inhibitors, scrambled oligonucleotides and control groups. The results from the Transwell migration assay revealed a significant difference in the experimental groups compared to the controls. Specifically, the upregulation of miR-224 significantly increased the number of cells that migrated through the membrane; in contrast, downregulation of miR-224 significantly inhibited the migratory ability of the HCC cells. In parallel, we analyzed the effect of miR-224 on HCC cell invasion using the Matrigel invasion assay. The results were similar to the migration assay mentioned above (Fig. 2).

$P P P 2 R 1 B$ is a candidate target of $m i R-224$. To explore the mechanism by which miR-224 regulates cell proliferation and metastasis, we performed a miRNA target search using PicTar and TargetScan. The 3'UTR of PPP2R1B was found to contain a highly conserved putative miR-224 binding site. In the present study, the level of endogenous miR-224 was up- or downregulated by transfection with a miR-224 mimic or miR-224 inhibitor, respectively; scrambled oligonucleotides were used as controls. Western blot analysis showed that the expression of PPP2R1B was elevated in HepG2 cells upon transfection of miR-224 inhibitor, relative to the control groups. Compared to the control groups, the expression of PPP2R1B was reduced in HepG2 cells upon transfection of miR-224 mimics. In addition, we generated the pPPP2R1B 3'UTR-luci-WT and pPPP2R1B 
A

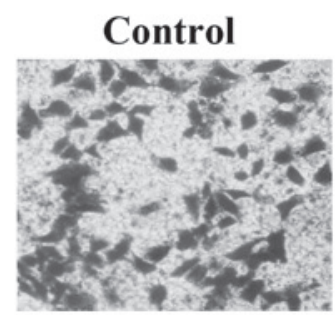

As-miR-224

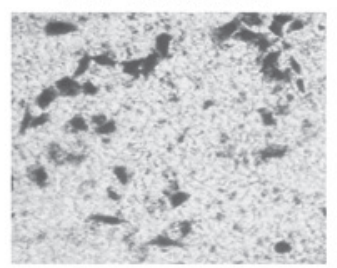

$\operatorname{miR-224}$

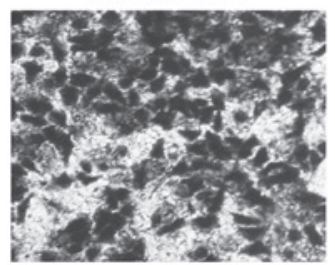

B

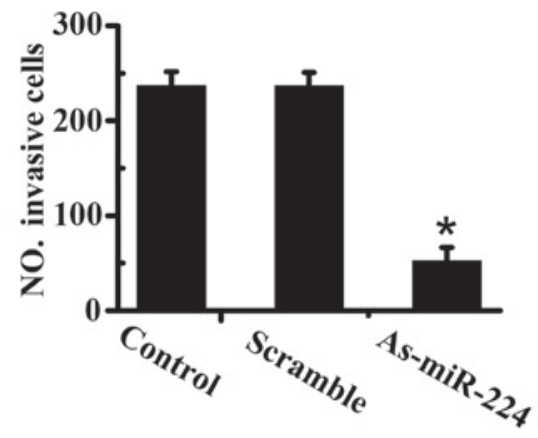

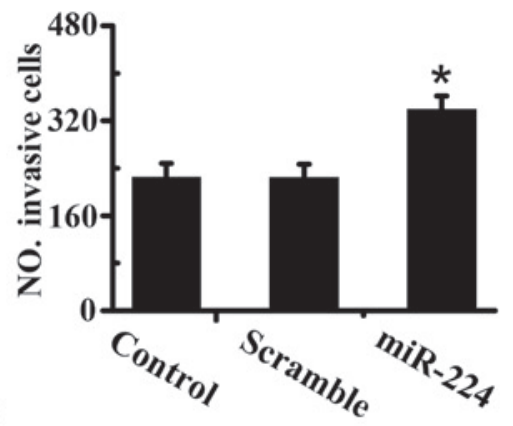

C

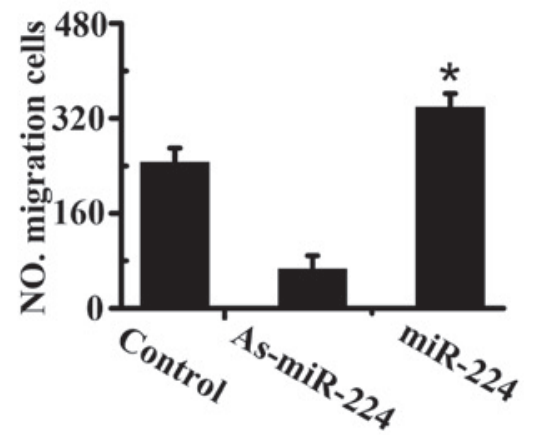

Figure 2. miR-224 promotes cell invasion and migration of HepG2 cells. Cell invasion and migration assays were carried out. Forty-eight hours after transfection, miR-224 inhibitors, miR-224 mimics, the two scrambled oligonucleotides and the control HepG2 cells were subjected to the Transwell assay, then incubated for another $36 \mathrm{~h}$ (for invasion assay) or $24 \mathrm{~h}$ (for migration assay). The number of cells stained with crystal violet was counted under a microscope in five random high-power fields. The average cell number was obtained from three independent experiments. (A) Representative images of the invasive cells fixed and stained on the membrane. (B) Average number of cells that migrated through the matrigel into the lower surface of the membrane in the different experimental groups. (C) Parallel results were obtained in the migration assay. Data are expressed as the mean $\pm \mathrm{SD}$. "P<0.05. As-miR-224, antisense miR-224.

A

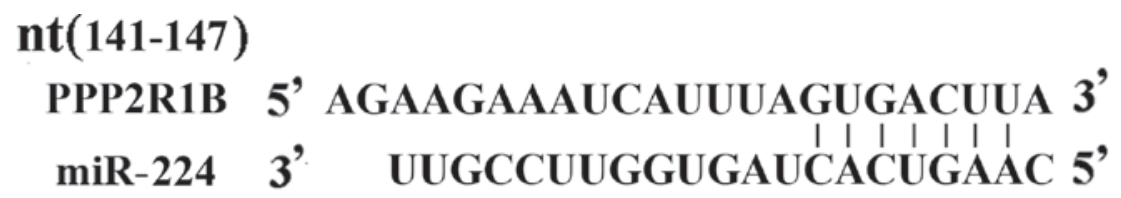

B
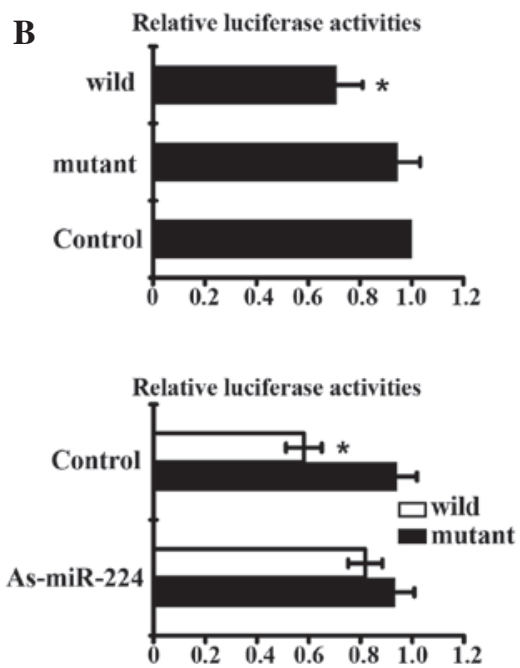

C

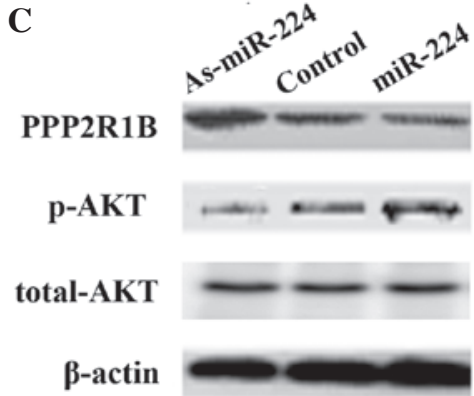

D

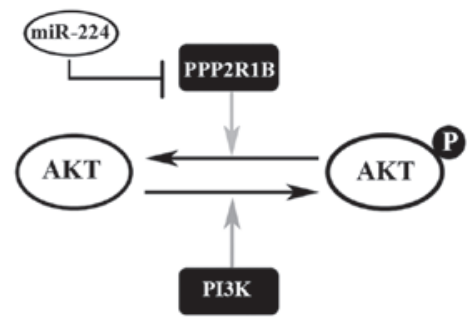

Figure 3. miR-224 modulates AKT signaling by targeting PPP2R1B. (A) Schematic representation of the putative binding sites in PPP2R1B 3'UTR for has-miR-224 seeding sequences. (B) Strong inhibition of luciferase activity was detected in HepG2 cells upon transfection of pPPP2R1B 3'UTR-luci-WT compared to cells transfected with either pPPP2R1B 3'UTR-luci-MUT or vector. (Bi) Firefly luciferase activity was standardized to Renilla construct. (Bii) Through downregulation of miR-224 levels with the miR-224 inhibitor, a clear increase in luciferase activity was observed in HepG2 cells upon transfection of pPPP2R1B 3'UTR-luci-WT, while no significant changes were detected in cells transfected with either pPPP2R1B 3'UTR-luci-MUT or vector. Columns, mean of three independent experiments; error bars, standard deviation. "P<0.05. (C) PPP2R1B, total AKT and phosphorylated AKT protein levels were detected by western blot analysis; protein levels were normalized to $\beta$-actin. The results suggested that miR-224 reduced the expression of PPP2R1B protein, and restoration of PPP2R1B was observed in miR-224 inhibitor-transfected HepG2 cells, compared with the control group. In addition, a reduction in phosphorylated AKT (but not total AKT) levels was detected in HepG2 cells treated with miR-224 mimics relative to the control group. (D) Schematic description of the possible molecular mechanism of miR-244 in HCC cells. As-miR-224, antisense miR-224. 
3'UTR-luci-MUT plasmids to investigate the interaction between miR-224 and its putative target sequences. Data from the luciferase reporter assay showed that the downregulation of miR-224 resulted in a marked increase in the luciferase activity of pPPP2R1B 3'UTR-luci-WT, without obvious changes in the luciferase activity of pPPP2R1B 3'UTR-luci-MUT. By contrast, the luciferase activity of pPPP2R1B 3'UTR-luci-WT decreased upon transfection of miR-224 mimics compared with the control groups. These results indicate that miR-224 directly modulates PPP2R1B expression by binding to the 3'UTR of PPP2R1B.

AKT signaling pathway is modulated by miR-224. We postulated that AKT signaling is modulated by miR-224, since PPP2R1B has been implicated in the negative control of kinase and phosphatase function, and therefore plays an integral role in numerous signal transduction pathways, including the AKT signaling pathway. To ascertain the effect of miR-224 on the regulation of the AKT signaling pathway, western blot analysis was performed to investigate the expression of AKT in the presence of miR-224. The results revealed that AKT phosphorylation was upregulated in HepG2 cells treated with miR-224 mimics, and downregulated in HepG2 cells upon transfection of miR-224 inhibitors compared to cells treated with negative control RNA sequences or transfection reagent alone. The expression of total AKT protein was also assessed, but there was no significant change upon transfection with miR-224 inhibitors or mimics. These data suggest that miR-224 impacts the AKT signaling pathway through modulation of AKT phosphorylation levels by regulation of PPP2R1B expression (Fig. 3).

Taken together, the data above suggest that miR-224 serves as a potent oncogenic microRNA in HCC progression by impacting cell proliferation, migration and invasion, possibly through the activation of AKT signaling by targeting PPP2R1B.

\section{Discussion}

Current data reveal that many miRNAs are frequently deregulated in human cancers (10), and play essential roles in cancer development and progression, as well as the invasionmetastasis cascade by targeting certain critical genes involved in HCC.

miR-224 is reported to be overexpressed in various types of cancer (15-20), and it plays critical roles in malignant phenotypes. Consistent with these previous observations, miR-224 was found to be upregulated in HepG2 cells in our study. Furthermore, in order to elucidate the precise function of miR-224, proliferation, migration and invasion assays were performed in HCC cells in the present study. Our results demonstrated that miR-224 positively regulates HCC cell proliferation, migration and invasion, which suggests that miR-224 might function in liver carcinogenesis and progression as an oncogenic regulator, although a previous study stated that miR-224 promotes HCC cell apoptosis by targeting the API-5 transcript (20). Despite great advances in determining that several deregulated miRNAs are involved in HCC, their precise roles in the malignant phenotypes of HCC cells are largely unknown, partly due to each miRNA having several putative targets with disparate functions.
To better understand the roles that miRNAs play in cells, certain pioneer researchers hypothesized that signal transduction pathways may be prime candidates for miRNA modulation, rather than individual putative genes (25).

In concordance with this theory, increasing data has shown that a number of aberrant miRNAs indeed modulate certain signaling pathways that have been extensively documented in HCC cells. In light of this recent evidence, we postulated that miR-224 might also impact certain key signaling pathways that are significant in $\mathrm{HCC}$.

In the present study, data from the western blot analysis revealed that the phosphorylation state of AKT correlated with miR-224 levels; more specifically, miR-224 appeared to activate AKT phosphorylation. In addition, since the levels of total AKT remained unchanged in the presence of miR-224 mimics and inhibitors, we reasoned that miR-224 modulates AKT activity in the process of phosphorylation.

AKT, also known as protein kinase B (PKB), is a serine/ threonine protein kinase that plays a key role in multiple cellular processes, and is associated with tumor cell survival, proliferation and invasion. The activation of AKT is also one of the most frequent alterations observed in human cancers (29). In fact, recent evidence suggests that a correlation exists between the AKT signaling pathway and deregulated miRNAs in human cancers, as mentioned above. In the present study, we demonstrated for the first time that miR-224 plays an essential role in regulating HCC cell functions, partly through modulation of the AKT signaling pathway, which most likely occurs via the inhibition of PPP2R1B, a putative target of miR-224.

PPP2R1B was selected as the focus of the study, as a result of analyzing the list of putative target genes identified by online software. PPP2R1B is a regulatory subunit of $\mathrm{PP} 2 \mathrm{~A}$, which is a conserved and ubiquitous serine/threonine phosphatase in eukaryotes. A previous study (22) suggested that PP2A functions as a suppressive regulator in various human cancers through the modulation of several oncogenic signaling cascades, including the AKT signaling pathway. Wong et al (10) suggested that the overexpression of miR-222 confers HCC cells with stronger metastasis abilities, through enhancing AKT signaling by targeting PPP2R2A, another subunit of PP2A.

More recently, Hamano et al (24) demonstrated that miR-200c is the responsible miRNA for chemoresistance in esophageal cancer. Furthermore, these authors showed that miR-200c-induced resistance is mediated through the activation of the AKT pathway, largely through the direct binding and suppression of PPP2R1B mRNA translation.

These two experiments suggested that PP2A might act as the important mediator between AKT signaling and aberrant miRNAs, which are able to inhibit the expression of the subunits that are indispensable for PP2A functions. In the present study, using a dual luciferase assay and western blotting, we demonstrated that miR-224 directly binds and inhibits PPP2R1B expression. Given that the reported role of $\mathrm{PPP} 2 \mathrm{R} 1 \mathrm{~B}$ is the negative regulation of AKT phosphorylation, we proposed that miR-224 indirectly regulates AKT signaling by targeting and suppressing PPP2R1B expression.

In summary, we demonstrated that miR-224 is upregulated in HepG2 cells, and the effects of miR-224 in HCC cells were also shown. Together, our data demonstrated that miR-224 
plays a role in the regulation of various cell functions, in addition to proliferation, cell migration and invasion. Moreover, we not only demonstrated that miR-224 functions as an oncogenetic regulator of $\mathrm{HCC}$ cells through the activation of AKT signaling, but also that the likely intermediary is PPP2R1B, which was identified as an miR-224 target in the present study. These results may lead to a better understanding of miR-224 function in $\mathrm{HCC}$, and also suggest a new direction for future studies aimed towards investigating aberrant miRNA functions in human cancers.

\section{Acknowledgements}

This study was supported by the China Postdoctoral Science Foundation (20080440910) and the Heilongjiang Provincial Health Office (2009-149).

\section{References}

1. Ferlay J, Shin HR, Bray F, Forman D, Mathers C and Parkin DM: GLOBOCAN 2008 v1.2, Cancer Incidence and Mortality Worldwide: IARC CancerBase No. 10. Lyon, France: International Agency for Research on Cancer, 2010.

2. Di Bisceglie AM: Issues in screening and surveillance for hepatocellular carcinoma. Gastroenterology 127: S104-S107, 2004.

3. Bartel DP: MicroRNAs: genomics, biogenesis, mechanism, and function. Cell 116: 281-297, 2004.

4. Bushati N, Cohen SM: MicroRNAs functions. Annu Rev Cell Dev Biol 23: 175-205, 2007.

5. Filipowicz W, Bhattacharyya SN and Sonenberg N: Mechanisms of post-transcriptional regulation by microRNAs: are the answers in sight? Nat Rev Genet 9: 102-114, 2008.

6. He L and Hannon GJ: MicroRNAs: small RNAs with a big role in gene regulation. Nat Rev Genet 5: 522-531, 2004.

7. Sayed D and Abdellatif M: MicroRNAs in development and disease. Physiol Rev 91: 827-887, 2011.

8. Calin GA and Croce CM: MicroRNAs signatures in human cancers. Nat Rev Cancer 6: 857-866, 2006.

9. Meng F, Henson R, Wehbe-Janek H, Ghoshal K, Jacob ST and Patel T: MicroRNA-21 regulates expression of PTEN tumor suppressor gene in human hepatocellular cancer. Gastroenterology 133: 647-658, 2007.

10. Wong QW, Ching AK, Chan AW, Choy KW, To KF, Lai PB and Wong N: MiR-222 overexprssion confers cell migratory advantages in hepatocellular carcinoma through enhancing AKT signaling. Clin Cancer Res 16: 867-875, 2010.

11. Li N, Fu H, Tie Y, Hu Z, Kong W, Wu Y and Zheng X: MiR-34a inhibits migration and invasion by down-regulation of c-Met expression in human hepatocellaular carcinoma cells. Cancer Lett 275: 44-53, 2009.

12. Salvi A, Sabelli C, Moncini S, Venturin M, Arici B, Riva P, Portolani N, Giulini SM, De Petro G and Barlati S: MicroRNA-23b mediates urokinase and c-met downmodulation and a decreased migration of human hepatocellular carcinoma cells. FEBS J 276: 2966-2982, 2009.

13. Tsai WC, Hsu PW, Lai TC, Chau GY, Lin CW, Chen CM, Lin CD, Liao YL,Wang JL, Chau YP, et al: MicroRNA-122, a tumor suppressor microRNA that regulates intrahepatic metastasis of hepatocellular carcinoma. Hepatology 49: 1571-1582, 2009.
14. Furuta M, Kozaki KI, Tanaka S, Arii S, Imoto I and Inazawa J: MiR-124 and miR-203 are epigenetically silenced tumor-suppressive microRNAs in hepatocellular carcinoma. Carcinogenesis 31: 766-776, 2010.

15. Prueitt RL, Yi M,Hudson RS, Wallace TA, Howe TM, Yfantis HG, Lee DH, Stephens RM, Liu CG, Calin GA, et al: Expression of microRNAs and protein-coding genes associated with perineural invasion in prostate cancer. Prostate 68: 1152-1164, 2008

16. Mees ST, Mardin WA, Sielker S, Willscher E, Senninger N, Schleicher C, Colombo-Benkmann $\mathrm{M}$ and Haier J: Involvement of CD40 targeting miR-224 and miR-486 on the progression of pancreatic ductal adenocarcinomas. Ann Surg Oncol 16: 2339-2350, 2009.

17. Arndt GM, Dossey L, Cullen LM, Lai A, Druker R, Eisbacher M, Zhang C, Tran N, Fan H, Retzlaff K, et al: Characterization of global microRNA expression reveals oncogenic potential of miR-145 in metastatic colorectal cancer. BMC Cancer 9: 374, 2009.

18. White NM, Chow TF, Mejia-Guerrero S, Diamandis M, Rofael Y, Faragalla H, Mankaruous M, Gabril M, Girgis A and Yousef GM: Three dysregulated miRNAs control kallikrein 10 expression and cell proliferation in ovarian cancer. Br J Cancer 102: 1244-1253, 2010.

19. Boguslawska J, Wojcicka A, Piekielko-Witkowska A, Master A and Nauman A: MiR-224 targets the 3'UTR of type 1 5'-iodothyronine deiodinase possibly contributing to tissue hypothyroidism in renal cancer. PLoS One 6: 24541, 2011.

20. Wang Y, Lee AT, Ma JZ, Wang J, Ren J, Yang Y, Tantoso E, Li KB, Ooi LL, Tan P and Lee CG: Profiling microRNA expression in hepatocellular carcinoma reveals microRNA-224 up-regulation and apoptosis inhibitor-5 as a microRNA-224-specific target. J Biol Chem 283: 13205-13215, 2008.

21. Li Q, Wang G, Shan JL, Yang ZX, Wang HZ, Feng J, Zhen JJ, Chen C, Zhang ZM, Xu W, et al: MicroRNA-224 is upregulated in HepG2 cells and involved in cellular migration and invasion. J Gastroenterol Hepatol 25: 164-171, 2010.

22. Ory S, Zhou M, Conrads TP, Veenstra TD and Morrison DK: Protein phosphatase $2 \mathrm{~A}$ positively regulates Ras signaling by dephosphorylating KSR1 and Raf-1 on critical 14-3-3 binding sites. Curr Biol 13: 1356-1364, 2003.

23. Basu S: PP2A in the regulation of cell motility and invasion. Curr Protein Pept Sci 12: 3-11, 2011.

24. Hamano R, Miyata H, Yamasaki M, Kurokawa Y, Hara J, Moon J, Nakajima K, Takiguchi S, Fujiwara Y, Mori M and Doki Y: Overexpression of miR-200c induces chemoresistance in esophageal cancers mediated through activation of the AKT signaling pathway. Clin Cancer Res 17: 2603, 2011.

25. Inui M, Martello G and Piccolo S: MicroRNA control of signal transduction. Nat Rev Mol Cell Biol 11: 2252-2262, 2010.

26. Datta J, Kutay H, Nasser MW, Nuovo GJ, Wang B, Majumder S, Liu CG, Volinia S, Croce CM, Schmittgen TD, et al: Methylation mediated silencing of microRNA-1 gene and its role in hepatocellular carcinogenesis. Cancer Res 68: 5049-5058, 2008.

27. Garofalo M, Di Leva G, Romano G, Nuovo G, Suh SS, Ngankeu A, Taccioli C, Pichiorri F, Alder H, Secchiero P, et al: miR-221 \& 222 regulate TRAIL resistance and enhance tumorigenicity through PTEN and TIMP3 downregulation. Cancer Cell 16: 498-509, 2009

28. Wang B, Hsu SH, Majumder S, Kutay H, Huang W, Jacob ST and Ghoshal K: TGFbeta-mediated upregulation of hepatic miR-181b promotes hepatocarcinogenesis by targeting TIMP3. Oncogene 29: 1787-1797, 2010.

29. Wendel HG, De Stanchina E and Fridman JS: Survival signalling by Akt and eIF4E in oncogenesis and cancer therapy. Nature 428: 332-337, 2004 